\title{
Estômatos em Briófitas e Pteridófitas. Alguns conceitos gerais
}

\author{
Eduardo Lleras (")
}

\begin{abstract}
Resumo
Alguns aspectos dos diversos tipos de estômatos nas Briófitas e Pteridófitas são discutidos aqui. As classificações morfológicas (Van Cotthem, 1970) e ontogenéticas (Kondo, 1962) são comparadas, visando a encontrar uma correlação entre as duas. Também são consideradas em detalhes as diversas hipóteses sobre a formação de estômatos "flutuantes".
\end{abstract}

\section{INTRODUÇÃo}

Neste trabalho visamos a apresentar uma comparação das classificações morfológicas $€$ ontogenéticas mais recentes dos estômatos de Briófitas e Pteridófitas, e a procurar encontrar uma correlação entre a classificação morfológica, segundo Van Cotthem, (1970) e ontogenética segundo Kondo (1962).

\section{ESTÔinATOS EM BRIÓFITAS}

Apesar de se esperar que este grupo vegetal tenha sido bem pesquisado em relação à origem e formação dos estômatos, isso, relativamente, há pouco tem sido feito.

O primeiro a observar a presença de estômatos em musgos (Musci) foi Shimper (1848, citado em Paton \& Pearce, 1957), que descreveu sua origem através de um levantamento de células epidérmicas já existentes. Em 1886, Vuillemin (citado em Paton \& Pearce, 1957) mostrou que a origem é igual à das plantas vasculares, através da divisão de uma célula-mãe em um estágio muito jovem do desenvolvimento da cápsula. Também indica que, no caso de Sphagnum, o desenvolvimento dos estômatos é incompleto. Também, em 1886, Haberlandt (citado em Paton \& Pearce, 1957) reportou a ausência de estômatos nas hepáticas (Hepaticae) com exceção da fase esporofítica de Anthoceros. Também descreveu a posição e forma dos estômatos em vários gêneros de musgos, explicando o mecanismo do movimento estomático.

Copeland (1902), no seu estudo sobre movimento estomático, estudou os gêneros Mnium e Funaria, nos musgos, e Anthoceros, nas hepáticas. Kuhlbradt, em 1922 (citado em Paton \& Pearce, 1957), baseado nas suas descrições de estômatos abortados, propôs uma relação filogenética entre Briófitas e Pteridófitas através de Anthoceros. Blaikley (1932) estudou as velocidades de transpiração de estômatos de Polytrichum.

A revisão de Paton \& Pearce (1957), sobre a ocorrência, estrutura e função de estômatos das Briófitas da Grã-Bretanha é, provavelmente, a mais completa $€$ útil, sobre estômatos de Briófitas, até esta data. Apesar de só incluir espécies presentes na Grã-Bretanha, pode ser consıderada válida, a nível genérico, para o resto do mundo. O artigo mais recente sobre o assunto é o Lindsay \& Finocchio (1970), o qual não acrescenta maior contribuição ao conhecimento da morfologia dos estômatos nos mus. gos.

\section{OCORRÊNCIA}

Nas Briófitas, os estômatos só ocorrem naturalmente na fase esporofítica. A maioria das cápsulas apresenta um colo que une o saco esporífero e a seta. Em alguns casos, este colo está mais desenvolvido, formando uma apófise sob a área esporífera da cápsula. Os estômatos geralmente se apresentam nesta apófise e no colo, regiões que tipicamente estão formadas por pequenas células fotossintéticas. Os estômatos podem apresentar-se distribuídos ao acaso ou formando anéis. Em algumas Polytrichaceae, os estômatos podem apresentar-se na ranhura formada entre a seta e a apófise.

(*) - Instituto Nacional de Pesquisas da Amazônia, Manaus. 
ONTOGENIA

O desenvolvimento dos estômatos começa quando o esporófito é ainda muito jovem. Certas células podem distinguir-se neste estágio por apresentarem cloroplastos maiores que as outras e por conterem amido. Durante o desenvolvimento inicial da cápsula, estas células dividem-se longitudinalmente; logo, a lamela media da parede mediana separa-se formando um poro. Estes poros são originalmente alo: gados (elípticos), porém, em alguns casos, dependendo do taxon, podem até sofrer encurtamento e tornarem-se circulares. Ao mesmo tempo que os poros estão sendo formados, pode-se observar um aumento no tamanho das células-guardas.

Normalmente, formam-se células-guardas, mas isto não é uma regra universal. Em alguns casos, só é formada uma célula-guarda, célula que, posteriormente, é rodeada pelas células acompanhantes. Isto pode ser freqüen. temente observado nas Hypnaceae. Em outros casos, células-guardas deste tipo podem ocorrer adjacentes a estômatos. Shrimper (1848, in Paton \& Pearce, 1957) descreveu uma célulaguarda solitária e circular para Funariaceae, um tipo que também é comum em Polytrichum e outros gêneros das Polytrichaceae.

Shimper descreveu o desenvolvimento destas células como inicialmente normal, mas com 0 aumento em tamanho dos estômatos, as paredes medianas não apresentam um cresci-

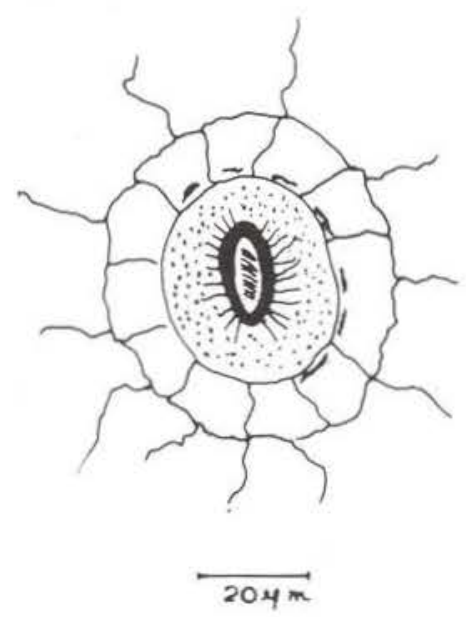

Figura 1. - Célula-guarda circular em Polytrichum, observação do autor.

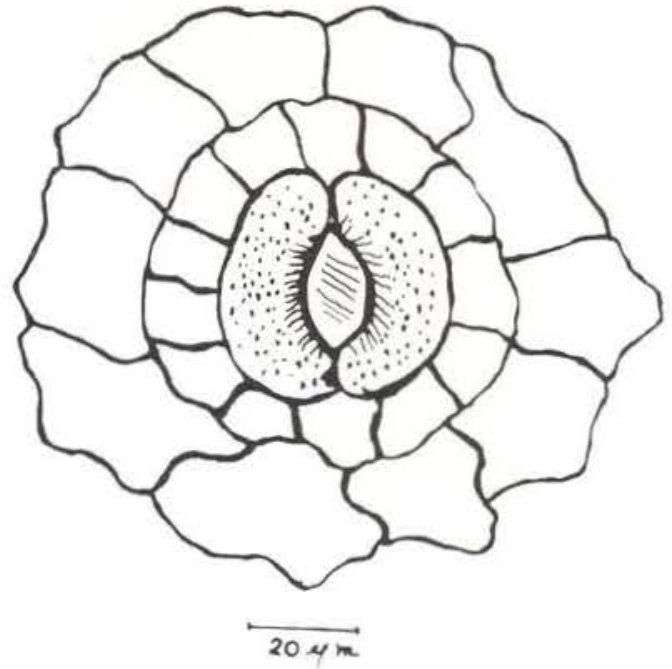

Figura 2. - Estômato de Polytrichum, mostrando a disposição actinocítica das células acompanhantes assim como o menor tamanho das mesmas.

mentc tão rápido como as paredes laterais, de maneira que as células-guardas permaneçam comunicadas entre si, formando uma célulaguarda anular binucleada. Haberlandt (1886). citado em Paton \& Pearce, (1957), sugeriu que no caso de Polytrichum, o desenvolvimento das paredes medianas é normal, com uma posterior reabsorção das mesmas.

Haberlandt também observou que a existência de estômatos formados por 3 ou 4 células-guardas é freqüente. Paton \& Pearce (1957), indicaram que isto é certo, mas consi. deram que são observações que não caracterizam taxon algum dentro das Briófitas, anotando que este tipo de absorção é encontrado em todos os grupos vegetais, quando as plantas são expostas a condições anormais de crescimento. No mesmo esporófito, é possível observar tanto estômatos normais (2 células) quanto estômatos formados por 3-4 células.

\section{MORFOLOGIA}

A classificação dos estômatos, de acordo com os tipos de células acompanhantes, indicanos que para as Briófitas são de dois tipos: Anomocítico ou Actinocítico, segundo a classificação proposta por Metcalfe \& Chalk (1950) e, mais tarde, modificada e completada por Van Cotthem entre 1968 e 1970. Em algumas espé- 
cies de Polytrichum, o tipo actinocítico é especialmente evidente, com as células acompanhantes dispostas radialmente ao redor das células-guardas, e de menor tamanho que estas

Em contraste com outros grupos vegetais nos quais os estômatos são geralmente meno. res que o restante das células epidérmicas, nos musgos o contrário é geralmente o caso (Ver fig. 2). O tamanho dos estômatos dado pela largura e comprimento de cada aparelho, quando observada a superfície, vai desde ca. $20 \times 20 \mu \mathrm{m}$, nos cases menores, até $60 \mu \mathrm{m}$ de largura $\times 80 \mu \mathrm{m}$ de comprimento no caso de Polytrichum formosum. Paton \& Pearce (1957) observaram que geralmente os estômatos pequenos tendem a apresentar poros circulares, sendo que estômatos grandes tendem a apresentar poros alongados (elipsóides).

Nas Briófitas, como em todo grupo vegetal que apresenta estômatos, o nível das célulasguardas em relação às restantes células ep:dérmicas pode ser de três tipos. A maioria dos musgos tem estômatos ao mesmo nível do resto da epiderme, ou emergentes muito pouco acima da mesma. Em muitas espécies de Polytrichum, Bryum, Funaria e outros, estão notavelmente acima do restante da epiderme. No caso de Sphagnum, podem estar elevados até doze ou mais células, o que produz um padrão típico na camada epidérmica. É de notar que os estômatos emergentes são geralmente grandes e apresentam poros compridos (Figs. 3A, 3B) .

O terceiro caso representa os estômatos que se encontram sob o nível da epiderme, variando desde ligeiramente imersos (ex. Dicra.

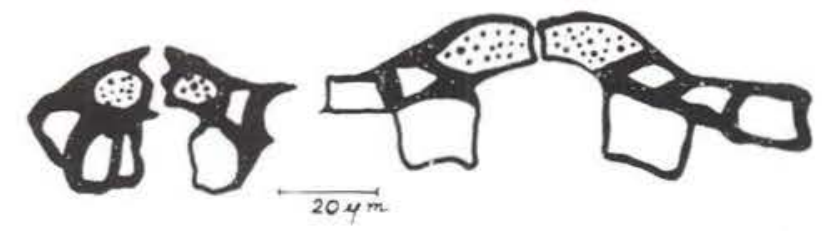

Figura 3. - Estômatos emergentes. a - Estômato de Polytrichum formosum em seção transversal da epiderme. b - Estômato de Aulacomnium palustre, seção transversal da epiderme. As duas figuras adaptadas de Paton \& Pearce (1957). nium scoparium) até situações nas quais os estômatos atingem o mesmo nível dos estratos subepidérmicos de células, como por exemplo, nas Mniaceae, certas espécies de Pohlia (Webera) e Hedwigia. Muitas espécies de Orthotrichium apresentam estômatos imersos com uma sobreposição parcial das células acompa. nhantes, formando assim uma câmara de ar entre o meio ambiente $e$ as células-guardas (Fig. 4, a, b) .
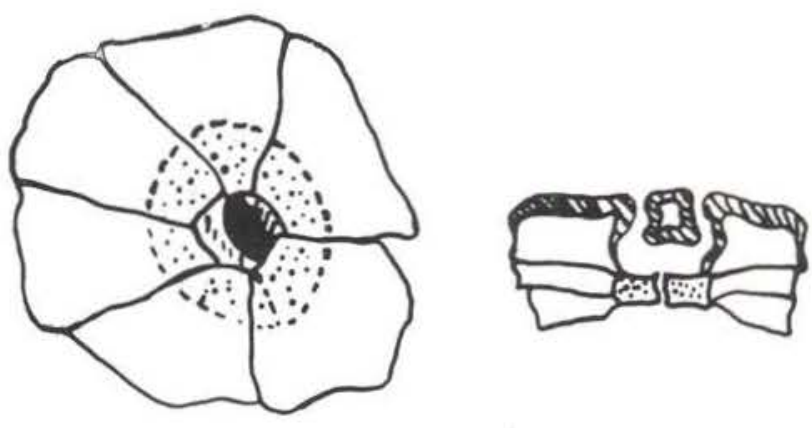

Figura 4 - a. Estômato de Ortotrichum sp em visão superficial; hipotético. b. Estômato de Ortotrichum anomale em seção transversal (segundo Paton \& Pearce (1957).

\section{SIGNIFICADO DA PRESENÇA DE ESTÔMATOS EM BRIÓFITAS}

A presença de estômatos nas Briófitas tem sido usada por muitos (ex. Smith, 1955) para postulá-los como provavelmente ancestrais de outros grupos vegetais considerados mais evoluídos. É um dos argumentos básicos dos defensores da teoria antitética de alternância de gerações para postular uma origem dentro das Briófitas para as Pieridófitas. Porém, os detensores da teoria isomórfica de alternância de gerações argumentam que, provavelmente, só indica um ancestral comum, provido de estômatos para os dois grupos (Briófitas e Pteridófitas). Smith (1955) indica os dois pontos de vista, mas sem favorecer algum deles. $\mathrm{Na}$ atualidade, não temos suficiente evidência para demonstrar de maneira inequívoca qual dos dois conceitos é certo.

Porém, dentro das Briófitas, os estômatos podem ter significado taxonômico bem grande, e ser assim úteis na compreensão da evolução 
do grupo. Apesar de já termos alguns trabaIhos a respeito, destacando-se o de Paton \& Pearce (1957), falta muito por fazer.

\section{ESTÔMATOS EM PTERIDÓFITAS}

O estudo de estômatos neste grupo é bem mais complexo que nas Briófitas, e uma grande quantidade de pesquisas está sendo realizada nele, entre outros por Van Cotthem, Kondo $\epsilon$ Mickel. O fato de os estômatos dos Pteridófitas terem despertado tanto interesse e provavelmente devido a que, como no caso das Angiospermas, os estômatos são um caráter taxonômico muito útil.

Sendo impossível, devido à grande quanti. dade de material disponível, considerar este grupo do mesmo modo com que foram discuti- das as Briófitas, esta seção estará limitada a discutir uma classificação de estômatos. Dois tipos de classificações principais têm sido postuladas: a de Kondo (1962), que é uma classificação ontogenética, e a de Van Cotthem (1970), que é uma classificação morfológica. Deve ficar claro aqui que estas duas maneiras de visualizar o problema não são contraditórias, mas complementam-se muito bem. Van Cotthem também apresenta alguns dados ontogenéticos no trabalho dele, mas é pouco o que tem para oferecer neste aspecto.

Tanto Van Cotthem quanto Kondo têm feito um levantamento bem compreensivo das Pteridófitas; mesmo assim não é totalmente completo. Eles têm examinado muitos gêneros de cada família, e apresentam dados para todas as famílias da classificaçăo segundo

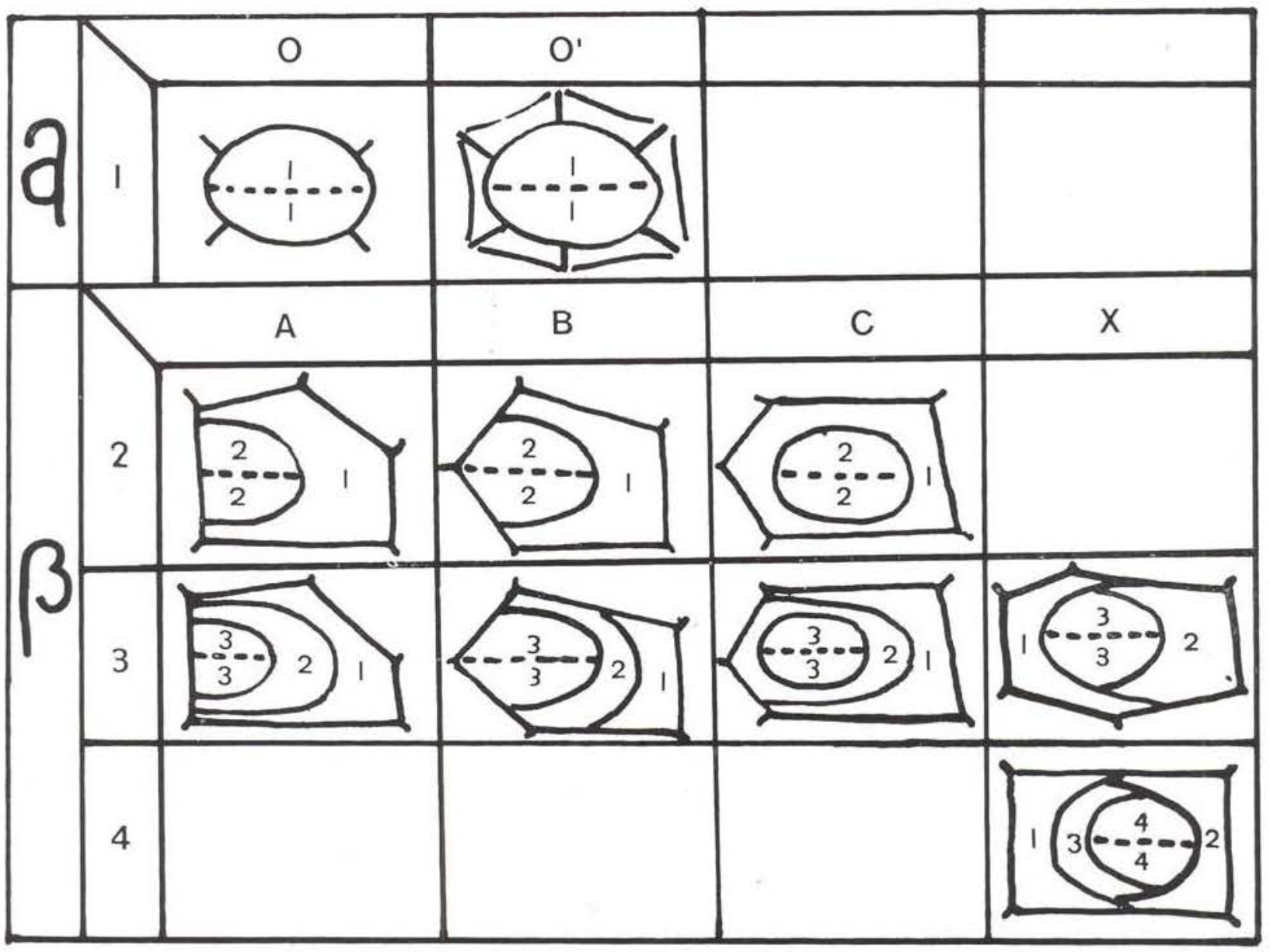

Figura 5 - Classificação ontogenética de Kondo (1962). Diagramática. Os números grandes 1, 2, 3, 4 da esquerda indicam o número de divisões das células iniciais; os números pequenos dentro das figuras indicam a ordem das divisões. 
Copeland (1947). As diferenças entre os dois em relação ao número de famílias só indica diferença de opiniões quanto à validez taxonômica de certos grupos, e não uma falta de pesquisa neles.

\section{CLASSIFICAÇÃO ONTOGENÉTICA}

Kondo (1962) considerou em detalhe a ontogenia de estômatos de Pteridófitas. Cons. tatou que todos os grupos estudados estão numa das categorias aqui relacionadas. Por conveniência, a classificação dele está traduzida aqui diretamente da publicação original de 1962.

A. Células-guardas dos estômatos desenvolvendo-se diretamente da célula-inicial dos estômatos .

A.1. Não se apresentam com divisões celulares fora das células estomáticas.

A.2. Apresentam-se com algumas divisōes fora das células estomáticas.

B. As células-guardas dos estômatos desenvolvem-se das células-iniciais diante de vá. rias divisões das iniciais.

B.1. As células-guardas desenvolvem-se através de divisões alternas da célula-inicial, e a direção do poro estomático é paralelo à célula-mãe.

A, B, C: As células-guardas desenvolvem-se através de divisões celulares unilaterais das células iniciais opostas às partes em crescimento da epiderme da fronde.

$A, B$ e C definem-se da seguinte ma. neira :

A. A primeira e seguintes iniciais dos estômatos desenvolvem-se unidas numa das paredes laterais da célula-mãe no lado do ponto de crescimento da folha.

B. A primeira e seguintes iniciais dos estômatos desenvolvem-se entre as duas paredes da céluiamãe no lado do ponto de crescimento da folha.

C. A última célula inicial desenvolve-se unida à porção interna da parede superior da célula-mãe.
Sendo as características da chave de Kondo um pouco confusas, é importante utilizar o quadro esquemático que ele apresenta para esclarecer os conceitos.

$\mathrm{Na}$ figura 5 apresentada, observa-se que, combinando o número de divisões com o padrão de desenvolvimento, apresentam-se dez padrões estomáticos nas Pteridóritas: 10, 10', $2 \mathrm{~A}, 2 \mathrm{C}, 3 \mathrm{~A}, 3 \mathrm{~B}, 3 \mathrm{C}, 3 \mathrm{X}$ e $4 \mathrm{X}$. Os outros padrões que faltam são hipoteticamente possíveis, mas não foram encontrados por Kondo em nenhuma das espécies estudadas.

\section{CLASSIFICAÇão MORFOLÓGICA}

Sendo impossível aqui fazer uma relação completa da "nova terminologia "de tipos estomáticos segundo Van Cotthem, apresenta-se aqui uma tabela indicando os principais tipos de estômatos propostos por ele. Nem todos eles se encontram nos fetos, mas a tabela 1 é útil para qualquer trabalho morfológico sobre estômatos .

Van Cotthem faz menção do desenvolvimento ontogenético no seu trabalho, mas com menos distinções quanto Kondo, a respeito. Baseados na classificação de Copeland, (1947) usada tanto por Kondo quanto Van Cotthem, tentamos aqui apresentando as famílias de fetos e tipos de estômatos segundo as duas classificações discutidas aqui, encontrar uma correlação entre os dois sistemas. Esta correlação tem, em muitos casos, provado ser impossivel de se estabelecer, como se pode observar na tabela 2.

Tanto Kondo quanto Van Cotthem, assim como Pant (1965) e outros, têm demonstrado que o conceito de Vesque (citado em Pant (1965)) de que a ontogenia pode ser facilmente determinada, estudando estômatos adultos não é sempre aplicável, sendo que duas estruturas idênticas podem ser formadas de diversos modos. Isto pode ser claramente observado, analisando a tabela N..$^{\circ}$. Vemos que é muito difícil correlacionarmos a estrutura de estômatos adultos com desenvolvimento ontogenético. É certo que alguns casos são bastante óbvios, mas isto não é certo na maioria dos casos. 


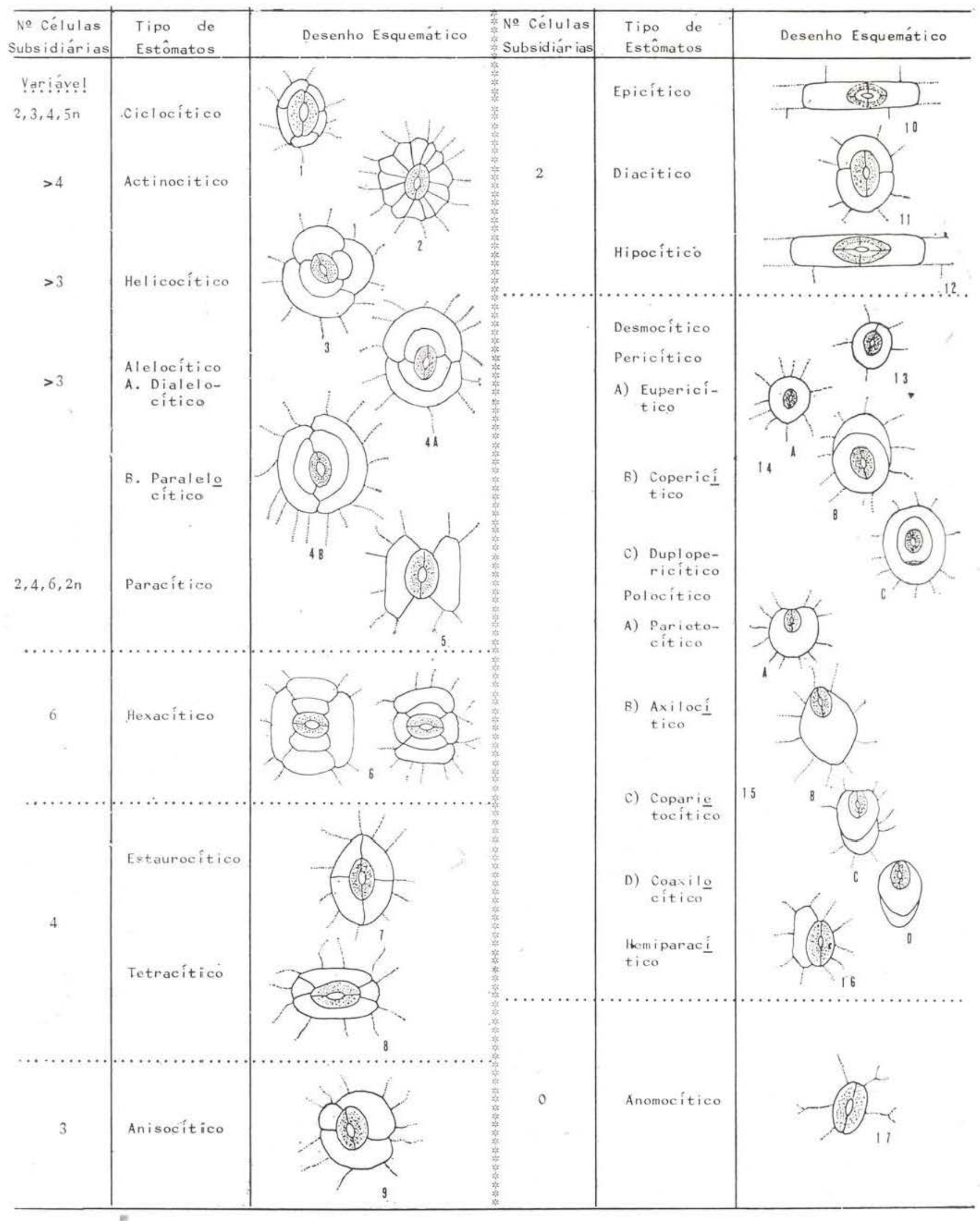

TABELA 1 - Classificação de estômatos segundo Van Cotthem, 1970. A nossa numeração nâo se encontra no trabalho original. 
Kondo considera os padrōes 10 e $10^{\prime}$ como primitivos. O tipo 10 está presente nas Ophioglossaceae e Osmundaceae, sendo que o tipo $10^{\prime}$ só está presente em Marratiaceae. Ophioglossaceae e Marratiaceae são fetos eusporangiados, sendo que Osmundaceae está classificada dentro dos leptosporangiados. Este sugere que as Osmundas são primitivas nesta característica, pelo qual Kondo sugere excluí-las dos leptosporangiados e estabelecer outra ordem ou sub-classe para acomodar a referida família.

Os tipos estomáticos $\mathrm{C}$ e $\mathrm{X}$ são restritos a certos grupos, e podem ser usados para indicar o grau de afinidade entre diversos grupos. Estes dois tipos são evidentemente especiali. zações dos tipos A e B. Os tipos A e B são mais intercambiáveis e recíprocos que os outros grupos assim como os mais comuns.

Segundo Van Cotthem, existem pelo menos nove tipos morfológicos de estômatos em fetos :

Polocitico : É o tipo mais comum, ocorrenao em 155 de 240 gêneros estudados; não está presente nas prı. meiras 6 famílias da tabela 2 com exceção dos gêneros Anemia e Mohlia, nas Schizaceae.

Anomocitico: Também muito freqüente, está presente em 85 gêneros; não está presente em Marratiaceae, Loxsomaceae ou Davalliaceae, e, nas Polypodiaceae, só nos fetos Gramniticos.

Ciclocitico: Presente só em Ophioglossaceae, Marratiaceae e Polypodiaceae.

Hipocitico: Presente só em Schizaceae e Pteridaceae.

Diacítico : Presente em Schizaceae, Gleichniaceae, Matoniaceae e Marsileaceae.

Pericitico : Em Schizaceae, Polypodiaceae e Vittariaceae.

Desmocitico: Presente em Schizaceae, Polypodiaceae e Vittariaceae.

Paracitico : Presente em loxsomaceae, Cyatheaceae, Aspleniaceae e Polypodiaceae.

Estaurocítico: Presente só em Davalliaceae e Aspidiaceae.
De acordo com a distribuição dos tipos es. tomáticos nas famílias de Pteridófitas, e menos dentro destas, Van Cotthem propõe aumentar o número de famílias para 31 , baseando-se nos tipos de estômatos. Também anota que o tipo polocítico deve ser considerado avançado, sendo que não ocorre nas famílias "mais primitivas". Também indica que o tipo paracítico é valioso dentro do ponto de vista taxonômico, sendo que indica e apoia afinidades de certos grupos, sugerindo possíveis linhas filogenéticas. Porém, considera que tipos estomáticos tais como raurocítico não têm valor taxonômico ao nível de família, e, provavelmente, representam linhas evolutivas laterais no esquema evolutivo.

Um caso especial, conhecido só das Pteridófitas, é o dos estômatos "flutuantes" ou segundo Van Cotthem, estômatos pericíticos. Várias interpretações têm sido dadas, através dos anos, à ontogenia deste tipo de estômatos . Hildebrand, em 1866 (in Mickel \& Lersten, 1967), concluiu que um estômato flutuante desenvolvia-se quando a célula apical de um tricoma bicelular modificado crescia através da célula basal, alcançando a parede oposta da célula basal, e, logo, desenvolvendo-se e dividindo-se para produzir duas células-guardas.

Strasburger (1867, citado em Mickel \& Lersten, 1967), trabalhando com Anemia, concluiu que as células-mães originavam-se em contacto lateral com mais de uma célula epidérmica. A parede formada era logo reabsorvida, deixando as células-guardas isoladas dentro de uma célula epidérmica.

Rauter, 1870 (citado em Mickel \& Larsten, 1967) reportou a célula-mãe como funiliforme; é dizer, uma célula que crescia "in situ" e era forçada sobre a superfície da epiderme pelo crescimento próprio e da célula epidérmicá envolvente, para logo dividir-se para formar duas células-guardas. Mickel \& Lersten (1967) apoiaram Rauter, apresentando evidência que demonstra a existência da célula-mãe funiliforme, e anotaram que ilusōes óticas poderiam ter causado o erro dos outros (fig. 6).

Ao comparar os resultados obtidos por Mickel \& Lersten (1967) com a classificação de Kondo (1962) - fig. 5, 2c-3c - observa-se 


Ophioglossaceae
Marratiaceae
Osmundaceae
Schizaceae
Gleichneriaceae
Pteridaceae
Parkeriaceae
Davalliaceae
Plagiogyriaceae
Cyatheaceae
Aspidiaceae
Blechnaceae
Aspleniaceae
Matoniaceae
Polypodiaceae
Vittariaceae
Marsileaceae

10

$10^{\prime}$

10

$2 \mathrm{~A}, 2 \mathrm{C}$

$2 \mathrm{~A}$

2A, 2X, 4X, 3A, 3B

3B

$2 \mathrm{~A}, 2 \mathrm{~B}, 3 \mathrm{~B}$

2B

$3 \mathrm{X}, 2 \mathrm{~B}, 3 \mathrm{~B}$

$2 \mathrm{~A}, 3 \mathrm{~A}, 2 \mathrm{~B}, 3 \mathrm{~B}$

$2 \mathrm{~A}, 2 \mathrm{~B}, 3 \mathrm{~A}$

2B, $3 \mathrm{~B}$

$2 \mathrm{~B}$

$2 \mathrm{~A}, 2 \mathrm{~B}, 2 \mathrm{C}, 3 \mathrm{~A}, 3 \mathrm{~B}, 3 \mathrm{C}, 3 \mathrm{X}, 4 \mathrm{X}$

2B, $3 \mathrm{~B}$
1,17

1

17

$11,12,13,14,15,17$

11,17

$7,12,15,17$

17

7,15

17

$5,15,17$

15,17

15,17

$7,15,17$

11,17

$1,5,13,14,15,17$

$13,14,15,17$

11,17 .

TABELA 2 - Tipo de estomatos presentes em famílias de fetos de acordo com Kondo e Van Cotthem. Para Kondo, a terminologia é a do artigo original dele; para Cotthem, os números correspondem àqueles dados por nós aos diferentes tipos estomáticos.<smiles>CC1CCC2CC3(C)CC4CC(C)C(C1)C4C23</smiles>

a

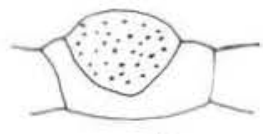

b

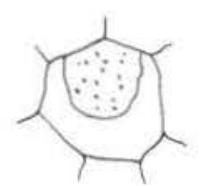

e
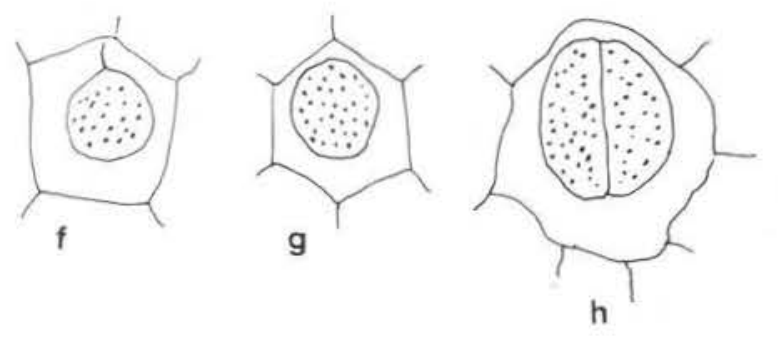

h

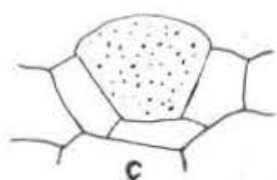

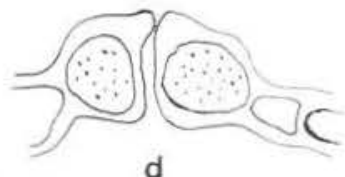

d

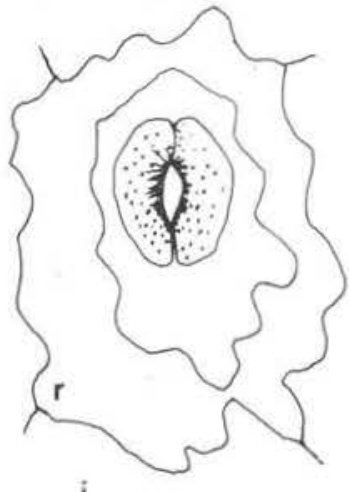

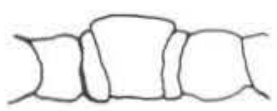

j

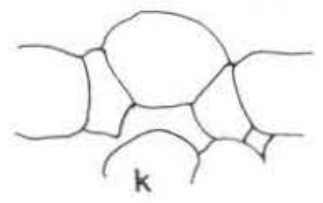

k

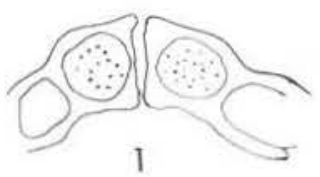

Figura 5 - Ontogênese dos estômatos flutuantes, segundo diversos autores. a-d, segundo Hildebrand; e-h, segıindo Strasburger; i, estômato flutuante adulto, segundo Mickel \& Lersten; j-l, segundo Rauter. Todos citados em Mickel \& Lersten (1967). 
uma diferença na ontogenia dos estômatos flutuantes. Para Kondo, são originados por uma divisão interna de uma célula epidérmica; é dizer a formação de uma célula no interior de outra. Este conceito é bem difícil de explicar ontogeneticamente. A explicação dada por Mickel \& Lersten é bem mais lógica e fácil de explicar ontogeneticamente, e a evidência foto. gráfica demonstra ser este o processo ontogenético correto.

\section{Summary}

A review was made of the most recent literature on morphology and ontogeny of stomata in Bryophyles and Pteridophytes. Comparisons were made between Kondo's (1962) ontogenetic and Van Cotthem's (1970) morphological classification of stomata, and possible correlations of the two systems were considered. It was concluded that there is no direct correlation, in most cases, between the ontogeny of stomata and the morphology of adult stomata. Floating stomata were also considered, and the current accepted ontogeny is discussed.

\section{BIBLIOGRAFIA CITADA}

BLAIKLEY, N.M.

1932 - The transpiration of the sporophytes of Polytrichum commune Anne. Bot. Lond., $46: 289$.

COPELAND, E.B.

1902 - Mechanism of stomata. Ann. Bot. Lond, $16: 327$
1947 - Genera Filicum. Chronica Botanica, Waltham Mass. 247 p.

Kondo, $\mathrm{T}$.

1962 - A contribution to the study of fern stomata. Res. Bull. Shizuoka Univ. Fac. Ed., $13: 239$.

LINDSAY, E. \& FinOCCHIO, A.F.

1970 - Stomate morphology of mosses. Science Studies, $26: 5$.

metcalfe, C.R. \& Chalk, L.

1950 - Anatomy of the Dicotyledons. Oxford, Clarendon Press.

MICKEL, J.T. \& LERSTEN, N.R.

1967 - Floating stomates (adestomy) in ferns: distribution and ontogeny. Amer. Jour. Bot., 54 : 1181.

PANT, D.D.

1965 - On the ontogeny of stomata and other homologous structures. Plant. Sci. Jour., $1: 1$.

Paton. J.A. \& Pearce, J.V.

1957 - The occurrence, structure and functions of stomata in British bryophytes. Trans. Brit. Bryol. Soc., 3: 228 .

SMITH, G.L.

SмIтн, G.M.

1955 - Criptogamic Botany. Vol. 2., 2.a Ed. Mc Graw-Hill, New York.

VAN COTTHEM, W.R.J.

1970 - A classification of stomatal types. Bot. Jour. Linn. Soc., $63: 235$.

1970 - Comparative morphological study of the stomata in the Filicopsida. Bull. Jard. Bot. Nat., Belg., $40: 81$. 